\title{
The intersection structure of $t$-intersecting families
}

\author{
John Talbot \\ Department of Mathematics \\ University College London, London, UK \\ talbot@math.ucl.ac.uk
}

Submitted: Jun 30, 2004; Accepted: Sep 24, 2005; Published: Oct 13, 2005

Mathematics Subject Classifications: 05D05, 05C65

\begin{abstract}
A family of sets is t-intersecting if any two sets from the family contain at least $t$ common elements. Given a $t$-intersecting family of $r$-sets from an $n$-set, how many distinct sets of size $k$ can occur as pairwise intersections of its members? We prove an asymptotic upper bound on this number that can always be achieved. This result can be seen as a generalization of the Erdös-Ko-Rado theorem.
\end{abstract}

\section{Introduction}

For integers $1 \leq r \leq n$ we define $[n]:=\{1,2, \ldots, n\}$ and $[n]^{(r)}=\{A \subseteq[n]:|A|=r\}$. In this short note we give an asymptotically sharp answer to the following extremal question: if $\mathcal{A} \subseteq[n]^{(r)}$ is $t$-intersecting and $t \leq k \leq r$ then how many distinct sets of size $k$ can occur as pairwise intersections of members of $\mathcal{A}$ ?

For example if $\mathcal{A} \subseteq[n]^{(6)}$ is 2-intersecting then how many 4-sets can occur as pairwise intersections of members of $\mathcal{A}$ ? (The answer in this case is at most $21\left(\begin{array}{l}n \\ 2\end{array}\right)+O(n)$ and this can achieved.)

Let $\mathcal{A} \subseteq[n]^{(r)}$ be $t$-intersecting and $t \leq k \leq r$. The family of $k$-intersections of $\mathcal{A}$ is

$$
\mathcal{A}\langle k\rangle=\left\{C \in[n]^{(k)}: \exists A, B \in \mathcal{A} \text { such that } A \cap B=C\right\} .
$$

If $\mathcal{A}$ is $t$-intersecting then trivially the smallest pairwise intersections of members of $\mathcal{A}$ have size $t$. These play an important role when trying to bound the size of $|\mathcal{A}\langle k\rangle|$ in general. In particular it is useful to define the following quantity for $1 \leq t \leq r$

$$
\alpha_{t}^{(r)}=\max _{n \geq r}\left\{|\mathcal{A}\langle t\rangle|: \mathcal{A} \subseteq[n]^{(r)} \text { is } t \text {-intersecting }\right\} .
$$

The fact that $\alpha_{t}^{(r)}$ is well-defined follows from a result of Lovász [2] which implies that $\alpha_{1}^{(m)}$ exists for all $m \geq 1$. It is easy to then check that $\alpha_{t}^{(r)} \leq \alpha_{1}^{m}$, for $m=\left(\begin{array}{l}r \\ t\end{array}\right)$, and so $\alpha_{t}^{(r)}$ exists for all $1 \leq t \leq r$. The following general upper bound for $\alpha_{t}^{(r)}$ was given in [3]. 
Theorem 1 If $1 \leq t<r$ then

$$
\alpha_{t}^{(r)} \leq \frac{1}{2}\left(\begin{array}{c}
2 r-t \\
r-t
\end{array}\right)\left(\begin{array}{l}
r \\
t
\end{array}\right)
$$

\section{Result}

Theorem 2 If $1 \leq t \leq k \leq r \leq n$ and $\mathcal{A} \subseteq[n]^{(r)}$ is t-intersecting then

$$
|\mathcal{A}\langle k\rangle| \leq \alpha_{t}^{(r-k+t)}\left(\begin{array}{c}
n \\
k-t
\end{array}\right)+O\left(n^{k-t-1}\right) .
$$

Moreover this bound can always be achieved.

Proof. We first prove the upper bound using induction on $k$. The result holds trivially for $k=t$ so suppose $k \geq t+1$. For $i \in[n]$ define

$$
\mathcal{A}_{i}^{\prime}=\{A \backslash\{i\}: i \in A \text { and } A \in \mathcal{A}\} .
$$

If $V=\bigcup_{A \in \mathcal{A}(t)} A$, then $|V| \leq t \alpha_{t}^{(r)}=O(1)$ by Theorem 1 (as $n$ tends to infinity). Note that if $i \in[n] \backslash V$ then $\mathcal{A}_{i}^{\prime}$ is a $t$-intersecting family of $(r-1)$-sets and hence by our inductive hypothesis for $k-1$

$$
\sum_{i \in[n] \backslash V}\left|\mathcal{A}_{i}^{\prime}\langle k-1\rangle\right| \leq \alpha_{t}^{(r-k+t)}\left(\begin{array}{c}
n \\
k-t-1
\end{array}\right)(n-|V|) .
$$

We now consider how often a set $A \in \mathcal{A}\langle k\rangle$ is counted in the left-hand side of (2). Partition $\mathcal{A}\langle k\rangle$ as $\mathcal{A}\langle k\rangle=\mathcal{B} \cup \mathcal{C}$, where

$$
\mathcal{B}=\{A \in \mathcal{A}\langle k\rangle:|A \cap V| \leq t\} \quad \text { and } \quad \mathcal{C}=\{A \in \mathcal{A}\langle k\rangle:|A \cap V| \geq t+1\} .
$$

If $A \in \mathcal{B}$ then $A$ is counted at least $k-t$ times in the left-hand side of (2) while

$$
|\mathcal{C}| \leq\left(\begin{array}{c}
|V| \\
k
\end{array}\right)\left(\begin{array}{c}
n-|V| \\
k-t-1
\end{array}\right)=O\left(n^{k-t-1}\right)
$$

Hence we have

$$
|\mathcal{A}\langle k\rangle| \leq \alpha_{t}^{(r-k+t)}\left(\begin{array}{c}
n \\
k-t
\end{array}\right)+O\left(n^{k-t-1}\right) .
$$

The upper bound then follows by induction on $k$.

The following simple construction shows that the upper bound (1) can always be achieved. Let $\mathcal{B}$ be a $t$-intersecting family of $(r-k+t)$-sets satisfying $|\mathcal{B}\langle t\rangle|=\alpha_{t}^{(r-k+t)}$. If $m=\left|\cup_{B \in \mathcal{B}} B\right|$ then we may suppose that $\mathcal{B} \subseteq[m]^{(r-k+t)}$. Let $n \geq m+k-t$ and consider the family

$$
\mathcal{A}=\left\{A \in[n]^{(r)}: \exists B \in \mathcal{B} \text { such that } B \subseteq A\right\} .
$$


Since $\mathcal{B}$ is $t$-intersecting so $\mathcal{A}$ is also $t$-intersecting.

To see that the family $\mathcal{A}$ gives equality in (1) consider any $k$-set $C=D \cup E$, where $D \in \mathcal{B}\langle t\rangle$ and $E \in\{m+1, m+2, \ldots, n\}^{(k-t)}$. By definition of $\mathcal{B}\langle t\rangle$ there exist $B_{1}, B_{2} \in \mathcal{B}$ such that $B_{1} \cap B_{2}=D$. Moreover $B_{1} \cup E, B_{2} \cup E \in \mathcal{A}$ and hence $C=D \cup E=$ $\left(B_{1} \cup E\right) \cap\left(B_{2} \cup E\right) \in \mathcal{A}\langle k\rangle$. Hence for each $D \in \mathcal{B}\langle t\rangle$ and each $E \in\{m+1, \ldots, n\}^{(k-t)}$ we obtain a unique set $C=D \cup E \in \mathcal{A}\langle k\rangle$. Thus

$$
\begin{aligned}
|\mathcal{A}\langle k\rangle| & \geq \alpha_{t}^{(r-k+t)}\left(\begin{array}{c}
n-m \\
k-t
\end{array}\right) \\
& =\alpha_{t}^{(r-k+t)}\left(\begin{array}{c}
n \\
k-t
\end{array}\right)+O\left(n^{k-t-1}\right) .
\end{aligned}
$$

The first part of this theorem then implies that equality holds in (1) for $\mathcal{A}$.

\section{Remarks}

The case $r=k$ of Theorem 2 says that if $\mathcal{A} \subseteq[n]^{(r)}$ is $t$-intersecting then

$$
|\mathcal{A}| \leq\left(\begin{array}{c}
n \\
r-t
\end{array}\right)+O\left(n^{r-t-1}\right)
$$

This is essentially a version of the Erdös-Ko-Rado theorem [1].

For $t=1$ we can prove a stronger result (see [4]). Namely, if $\mathcal{A} \subseteq[n]^{(r)}$ is intersecting then either $\mathcal{A}$ is constructed as in the example (3) given above, or $\mathcal{A}$ has far fewer $k$ intersections than this example.

For the bound in Theorem 2 to be explicitly calculated we need to know the value of $\alpha_{t}^{(r-k+t)}$. This is an important problem in its own right. The values that are currently known are: $\alpha_{t}^{(t)}=1, \alpha_{t}^{(t+1)}=\left(\begin{array}{c}t+2 \\ 2\end{array}\right), \alpha_{1}^{(3)}=7, \alpha_{1}^{(4)}=16$ and $\alpha_{2}^{(4)}=21$. An upper bound for $\alpha_{t}^{(r)}$ is given by Theorem 1 while the best known bounds for $\alpha_{1}^{(r)}$ are due to Tuza [5].

Theorem 3 (Tuza [5]) If $r \geq 4$ then

$$
2\left(\begin{array}{c}
2 r-4 \\
r-2
\end{array}\right)+2 r-4 \leq \alpha_{1}^{(r)} \leq\left(\begin{array}{c}
2 r-1 \\
r-1
\end{array}\right)+\left(\begin{array}{c}
2 r-4 \\
r-1
\end{array}\right) .
$$

\section{References}

[1] P. Erdős, C. Ko and R. Rado, Intersection theorems for systems of finite sets, Quart. J. Math. Oxford (2) 12 (1961), 313-320.

[2] L. Lovász, "Combinatorial Problems and Exercises", North Holland, Amsterdam, New York, Oxford, 1979.

[3] J. Talbot, A new Bollobás-type inequality and applications to $t$-intersecting families of sets, Discrete Math. 285, 1-3, (2004), 349-353. 
[4] J. Talbot, The number of $k$-intersections of an intersecting family of $r$-sets, $J$. Combin. Theory Ser. A, 1062 (2004), 277-286.

[5] Zs. Tuza, Critical hypergraphs and intersecting set-pair systems, J. Combin. Theory Ser. B, 39 (1985), 134-145. 